\title{
Energy-Efficient Self Reconfigurable Clustering Approach for Heterogeneous Wireless Sensor Networks
}

\author{
M.Jagadeeswara Reddy \\ Associate Professor \\ Ravindra College of engineering \\ for women \\ Kurnool,AP,INDIA.
}

\author{
P.Chenna Reddy, PhD. \\ Associate Professor, HOD of \\ CSE, JNTUA college of \\ engineering Pulivendula, \\ Pulivendula
}

\author{
A.Ganesh \\ Assistant Professor \\ Ravindra College of engineering \\ for women \\ Kurnool,AP,INDIA
}

\begin{abstract}
Self Reconfigurable Clustering (Re-Clustering) mechanism is very useful and emerging technique whenever the cluster head failure occurs in the network due to limited battery power, low radio transmission range, minimum memory speed. The energy compulsion of each sensor node directly affects the scalability, efficiency and lifetime of a sensor network. In heterogeneous wireless sensor network, each node is having different amount of energy. This paper mainly focuses on Reclustering in heterogeneous wireless sensor network for maintain the load balance and data aggregation. The proposed protocol mainly focus on the key parameters of the sensor nodes which are prolong the network lifetime such as average residual energy of the each cluster head in order to increase the network lifetime, energy dissipation of the sensor nodes.
\end{abstract}

Keywords: Re-clustering, heterogeneity, Load balancing, Data aggregation

\section{INTRODUCTION}

Recent advances in wireless communications technology and MEMS (Micro-electronic-management-systems) and low power and integrated digital electronics have enabled the development and deployment of low energy, tiny, microsensors in a Wireless sensor Networks (WSN) [1-5]. WSN's have been widely concerned as one of the important emerging technologies for current century [6][8]. These huge no of sensors are distributed anonymously to detect and monitor physical or environmental conditions such as vibration, pressure, temperature, sound and provides opportunities for a wide variety applications like as battle field monitoring, civilian and military applications [7]. Initially wireless sensor networks are motivated by military applications such as battle field monitoring. However these networks are now used in many application areas wild animal monitoring, home automation, health care applications, and even for commercial applications such as target tracking and so on. For all these civilian applications the data gathering is the main concern able aspect.

Thus the elementary goal of WSN is to produce crude local data obtained (Sensed data) by every sensor node increase the network span of WSN as much as possible. The limited energy of sensor node mandates the design of clustering.

The researchers have worked on different aspects of wireless sensor networks like routing, data dissemination \& fusion, QOS, MAC, etc. Energy efficiency is one of the crucial aspects to increase the life time of the sensor network.

We considered the cluster based wireless sensor networks to improve the scalability of MAC as well as routing process.
Cluster heads works as a fusion spots for data aggregation, so the traffic for data transmission to base station is reduced. Clustered wireless sensor networks are divided in to two namely homogeneous and heterogeneous [9].

In homogenous environment all the sensor nodes have a equal level of battery power, so once one node is selected as cluster heads it should worked until it's life time over. So it is over loaded and every node is picked as a cluster head randomly and more over extra processing is also required to process data aggregation and protocol coordination [LEACH] [10] perform really well in homogeneous conditions.

On the other hand in heterogeneous wireless sensor networks node have different energy level and different functionalities. So to incorporate the data transmission via cluster heads and efficient hardware design is still crucial research issue in the field of heterogeneous wireless sensor networks [9].

The method data aggregation (transmits the data via cluster heads to base station and control the redundant transmissions) can effectively reduce the battery dissipation to achieve the primary goal of the wireless sensor network that is 'energy'. Especially for this data aggregation more widely used to different aggregation scenarios namely chain based and cluster based [11].

In cluster based scenario, many nodes are elected as a cluster heads and rest of the cluster are joining appropriately to form a cluster. After framing cluster, cluster heads are collected sensed data from its cluster members and transmit to back-end base station or sink node. In this concern when we reduce the no. of transmission it achieves energy efficiency. But this not so easy why because the cluster heads are dies faster. Factors affecting quick die of the sensor nodes are:

1. Cluster heads are performing serveral operations than cluster members so automatically cluster heads looses more energy.

2. Cluster size can also affect the load balance (i.e. if the no. of cluster nodes (scalability) are increased under one cluster head).

3. Distance from the base station also considerable.

Once the death of cluster head happens, the large no. of control packets should be sent in order to reselect the new cluster heads and construct new cluster requires some more energy dissipation and increases load.

Now a days the sensors are used for monitoring different areas and data, sensor nodes are set to be different energies in order to monitor data in different times is called heterogeneous wireless sensor network. In this type of network the energy based re-clustering mechanism is more useful to reduce more battery dissipation and achieve load balance. 


\section{RELATED WORK}

In most of the previous research Energy dissipation caused by the factors like Additional energy dissipated for data retransmission Due to load of data aggregation Transmission distance. In this concern for factor I the researchers are proposed to main a backup cluster heads. Backup cluster heads are selected at the time cluster head selection and informed to all of the cluster members, if the cluster heads residual energy if less than the threshold value it is informed to the backup cluster head to take care about the control packets. Here backup cluster heads are common node and transmits the sensed data to the cluster head until it becomes cluster head [12]. If the load of data aggregation (factor II) is two excessive, LEACH $[10,13]$ uses the probability value to determine the cluster heads in each round. In this cluster heads are not selected via base station so it reduces the excess delay. For factor III(transmission distance ) EECCRS[14] improved network load unbalanced which differentiate hierarchal areas using concentric circles. In this a chain based infrastructure and residual energy is a key concern.

\section{ENHANCED ENERGY-AWARE RE- CLUSTERING}

In this section we discussed about the basic energy model for sensor network, about heterogeneous network and the proposed scheme i.e. re-clustering process

\subsection{Energy model}

Consumption of energy is achieved in WSN is two parts, consuming energy for computing, processing and collected data transmission. we have consider the energy model.Fig.1 which is already proposed in [10]

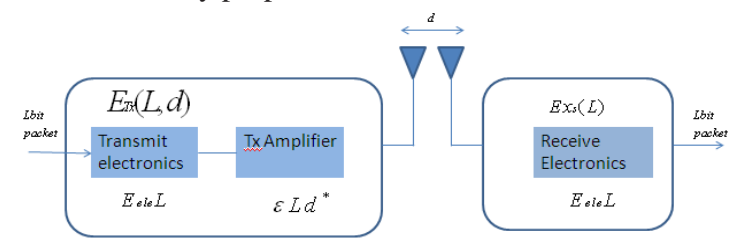

Fig 1: Radio energy dissipation model

Based on radio dissipation model in order to achieve an acceptable signal noise in transmitting $\mathrm{M}$ bit message over a distance $d$, energy expanded by radio is given by

$$
\begin{gathered}
E_{T x}(M . D)=M \cdot E_{\text {elec }}+M \cdot \varepsilon_{f s .} d^{2} \text { if } \mathrm{d} \leq d_{o} \\
=M \cdot E_{\text {elec }}+M \cdot \varepsilon_{f s .} \cdot d^{4} \text { if } \mathrm{d} \geq d_{o}
\end{gathered}
$$

The energy consumption of a sensor node receiving $\mathrm{m}$ bits data is

$$
\boldsymbol{R}_{x}(m)=m \cdot \text { Eelec }_{\text {el }}
$$

Where $E_{\text {elec }}$ is the energy dissipated per bit to run transceiver circuit. $\boldsymbol{E}_{f s}$ and $\boldsymbol{\varepsilon}_{m p}$ is depends on transmitter amplifier model, and $\mathrm{d}$ is the distance. This energy model is constructed up to transmission models free space and multi-path fading models. if the distance is less $\left(d_{o}\right)$ free space model is $\operatorname{applied}\left(d^{2}\right)$ and if $\mathrm{d}$ is greater than $\left(d_{o}\right)$ multipath fading is $\operatorname{applied}\left(d^{4}\right.$ energy consumed) . To receive an $\mathrm{M}$ bit of message the radio allocate

$$
E_{R x}=M \cdot E_{\text {elec }}
$$

\subsection{Proposed Re-Clustering Model:-}

In this section we discussed about heterogeneous wireless sensor networks and how the self adjustable clustering occurs.

\subsubsection{Heterogeneous wsh}

In this proposed paper we are constructed heterogeneous wireless sensor network have a different transmission ranges with same initial energy level. All the nodes in the sensor network are randomly distributed .Fig.1. illustrates the network environment.

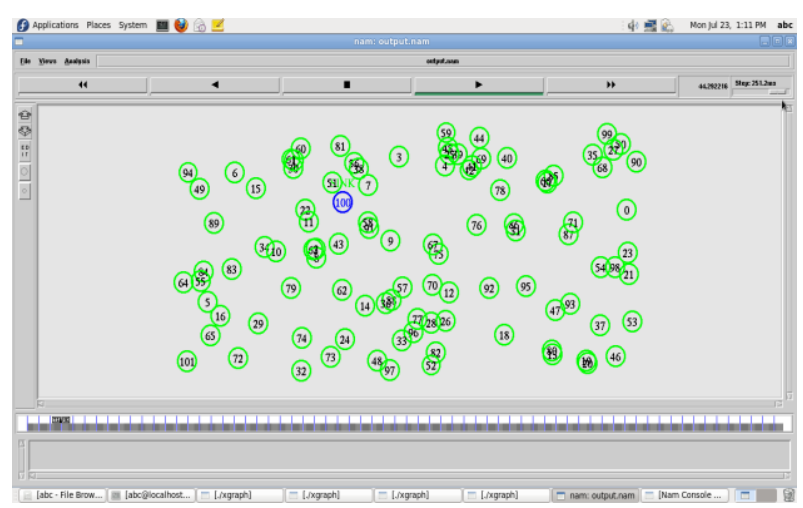

Fig 2: Heterogeneous WSN

\subsubsection{Re-Cluster formation process}

The enhanced energy-aware Re-clustering uses the residual energy is a main metric for the cluster head to determine the reselect of the cluster heads and also defines the residual energy threshold ( $E_{\text {Thre }}$ ) of the each cluster head. For setting the energy threshold of the cluster head we use residual energy of the cluster head is used to compute the node's energy dissipation percentage. The re-clustering should be done whenever the residual energy of the current cluster head is less than the threshold.

$$
E_{\text {Thre }}=E_{x} *\left(D_{x, b s} / D_{\operatorname{Max}}\right) *\left(C C_{x} / C C_{a v g}\right)
$$

Where $E_{x}$ is the residual energy of node $x$ as the cluster head and $D_{x, b s}$ is the distance between node to base station, $D_{\text {Max }}$ is the maximum distance from the base station to the network, $C C_{x}$ is the communication cost between $\mathrm{x}$ and other rest of nodes in the cluster. $\boldsymbol{C C}_{\text {avg }}$ is the average communication cost between the cluster nodes.

$$
C C_{\text {avg }}=\sum_{x=1}^{M} T C_{\cos t} / M
$$

Where $\mathrm{M}$ is the no. of nodes belongs to cluster and $T C \cos t$ is the total communication cost of the nodes in the cluster.

Re-clustering is a automatic process implemented in the protocols agent file triggered when the cluster head residual energy is less than the threshold energy of the node.reclustering is not require when the cluster head have energy greater than threshold value. Re-clustering mechanism decides the cluster head replacement in each round until all the sensor nodes die.

\section{SIMULATION SETUP}

In the simulation, environment consisted 100 sensor nodes randomly deployed in the area $100 \mathrm{~m} \mathrm{X} 100 \mathrm{~m}$ with initial 
energy 0.5 Joules. The sink node is coordinated at $(75,180)$.For all sensor nodes transmission frequency set randomly with communication range $50 \mathrm{~m}$.the size of the data packet, control packet as 2000, 1000 respectively. The overall simulation process is conducted for average residual energy of the cluster head and, network lifetime, energy dissipation of the network. Fig.3, Fig 4 illustrates the no of nodes span is compared with no. of rounds. In the existed approaches no. of alive nodes are quickly decreased rather than proposed solution,, Fig.5 and Fig.6 illustrate the comparison of energy dissipation, residual energy with no of rounds. The existed heterogeneous techniques, the proposed simulation results show very less dissipation. The Performance of the LEACH is good for homogeneous environments but not for heterogeneous conditions and not fit for large scale or very small clustering, so the proposed approach is better than the Existed scenarios with no. of clusters.

Table 1. Illustarates the Comparison table shows the performance of existed and current techniques in different environmental conditions

Table 1.Comparison table for Homogeneous and Heterogeneous conditions

\begin{tabular}{|l|l|l|l|l|l|l|}
\hline Solo & Technique & Environment & Rounds & $\begin{array}{l}\text { Network } \\
\text { Life time }\end{array}$ & $\begin{array}{l}\text { Residual } \\
\text { energy }\end{array}$ & $\begin{array}{l}\text { Energy } \\
\text { Dissipatio } \\
\text { n }\end{array}$ \\
\hline 1 & \multirow{2}{*}{ LEACH } & Homogeneous & 1000 & Excellent & Good & Low \\
\cline { 3 - 7 } & & Heterogeneous & 1000 & Good & Good & High \\
\hline 2 & \multirow{2}{*}{$\begin{array}{l}\text { Existed } \\
\text { Schemes }\end{array}$} & Homogeneous & 1000 & Excellent & Good & Low \\
\cline { 3 - 7 } & Heterogeneous & 1000 & Good & Good & High \\
\hline 3 & $\begin{array}{l}\text { Proposed } \\
\text { Approach }\end{array}$ & Homogeneous & 1000 & Excellent & Good & Low \\
\cline { 3 - 7 } & Heterogeneous & 1000 & Excellent & Excellent & Low \\
\hline
\end{tabular}

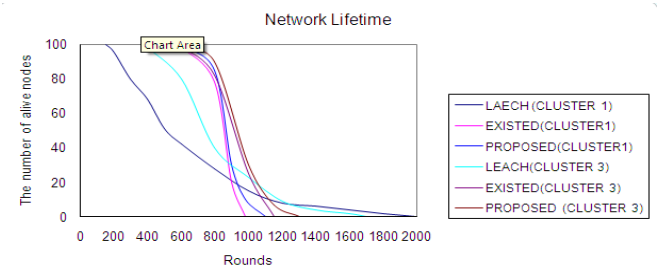

Fig 3: .Network life time with 1cluster vs. 3 clusters

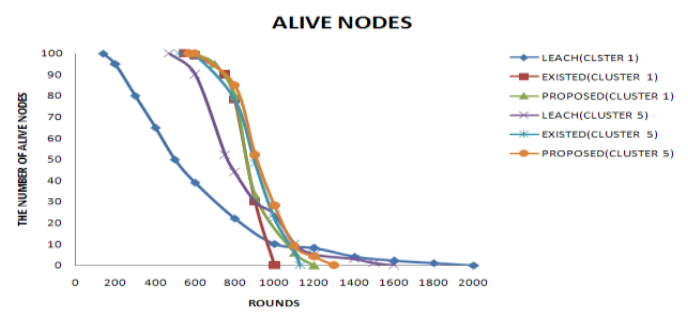

Fig 4: Network life time with 1cluster vs. 5 clusters

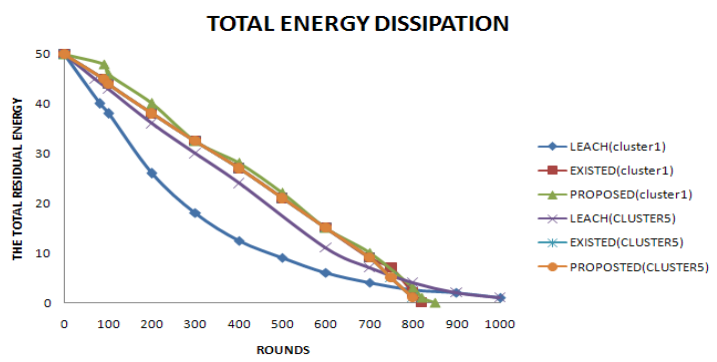

Fig 5: Energy Dissipation with 1 cluster vs. 3 clusters

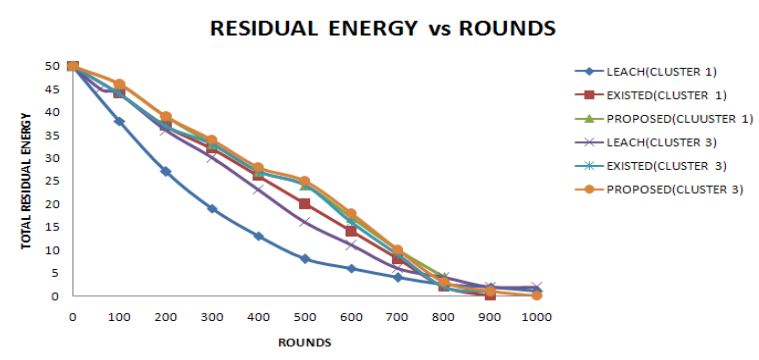

Fig: 6: Energy Dissipation with 1 cluster vs 5 clusters

\section{CONCLUSION}

In heterogeneous wireless sensor network link stability is key issue due to different data transmission frequencies and high energy dissipation of the sensor node, our proposed work address this key issue by calculated average residual energy of the cluster head with concern data transmission frequencies and ratio of transmissions to sink node. Simulation mainly evaluated the network performance in terms of energy dissipation of the sensor node variation with no. of clusters formed in the network. Cluster head failures happened slightly less than existed work. The no. of alive nodes are increased with no. of rounds. The proposed simulation results shows the high energy load balance ,efficient data aggregation and low energy dissipation of the node, which prolongs the network life time in the homogeneous wireless sensor networks.

\section{REFERNCES}

[1] Akyildiz, I.F. 2002 Wireless sensor networks: a survey, Computer Networks 393-422.

[2] Sohrabi, K. 2000 Protocols for self-organization of a wireless sensor network, IEEE Personal Communications 16-27.

[3] Min, R. 2001 Low power wireless sensor networks, in: Proceedings of International Conference on VLSI Design, Bangalore, India, January

[4] Rabaey, J.M. 2000 PicoRadio supports ad hoc ultra low power wireless networking, IEEE Computer 33 (7) 4248.

[5] Katz, H. and Kahn, J.M. Pister, K.S.J. 1999 Mobile networking for smart dust, in: Proceedings of the 5th Annual ACM/ IEEE International Conference on Mobile Computing and Networking (MobiCom_99), Seattle, WA, August.

[6] 21 ideas for the 21st century. 1999 Business Week, Aug. 30, pp. 78-167.

[7] Chong, C.Y. and Kumar, S.P. 2003.Sensor Networks: Evolution, opportunities, and Challenges Proceedings of the IEEE, vol. 91, no. 8, Aug., pp.

$1247-1256$

[8] Jun Zheng and Abbas Jamalipour, 2009.Wireless Sensor Networks: A Networking Perspective., a book published by A John \& Sons, Inc, and IEEEE,

[9] Vivek Mhatre and C.Rosenberg, Homogeneous vs Heterogeneous Clustered Sensor Networks: A Comparative Study Purdue University, West Lafayette, IN 47907-1285.

[10] Heinzelman, W. and Chandrakasan, A. and Balakrishnan, H. 2002 \An Application-Speci- c Protocol 
Architecture for Wireless Microsen- sor Networks," IEEE Transactions on Wireless Communications, Vol. 1,

[11] An Energy-aware Re-clustering Algorithm in Heterogeneous Wireless Sensor Networks by Hung- Chi Chu1, Meng-Hung Chi1.

[12 ]Hashmi, S. U. Mouftah, H. T. Georganas, N. D. 2007 Achieving Reliability Over Cluster-Based Wireless Sensor Networks Using Backup Cluster Heads, Global Telecommunications Conference, pp. 1149- 1153, Nov.
[13] Heinzelman, W. R. Chandrakasan, A. and Balakrishnan, H. 2000 Energy- Efficient Communication rotocol for Wireless Microsensor Networks The 33rd Annual HawaiiInternational Conference on System Sciences, Vol. 2, pp. 1-10, Jan.

[14] Park, M. W. , Choi, J. Y. Han, Y. J. and Chung, T. M. 2009 An Energy EfficientConcentric Clustering Scheme in Wireless Sensor Networks," Fifth International JointConference on INC, IMS and IDC, pp. 58-61. 